\title{
Evaluation of bioadhesive capacity and immunoadjuvant properties of vitamin $B_{12}$-Gantrez nanoparticles
}

Hesham H. Salman ${ }^{1}$, Carlos Gamazo ${ }^{2}$, P. Chris de Smidt ${ }^{3}$, Gregory Russell-Jones ${ }^{4}$, Juan M. Irache , $^{\text {,* }}$

${ }^{1}$ Centro Galénico, ${ }^{2}$ Departamento de Microbiologia, University of Navarra, Ap. 177, 31080-Pamplona, Spain.

${ }^{3}$ Parnassia Research Centre, Monsterseweg 83, 2553 RJ Den Haag. The Netherlands. Tel.: +31-703916624; fax: +31-703916612

email: c.de.smidt@parnassia.nl

${ }^{4}$ Apollo Life Sciences, 147 Queen Street, Beaconsfield, Sydney, NSW 2015, Australia. Tel.: +612 94171437; fax: +61294170681

email: contact@apollolifesciences.com

\author{
*Corresponding Author: \\ Juan Manuel Irache \\ Facultad de Farmacia \\ University of Navarra \\ Apartado. 177. 31080 Pamplona. España \\ Tel: 0034-948-42-5600, Ext: 6313 \\ Fax: 0034-948-425649 \\ E-mail: jmirache@unav.es
}




\section{Abstract}

PURPOSE: To design bioadhesive Gantrez AN (poly[methyl vinyl ether-co-maleic anhydride], PVM/MA) nanoparticles (NP) coated with Vitamin $\mathrm{B}_{12}$ (Vit $\mathrm{B}_{12}$ ), and investigate their application in oral antigen delivery.

METHODS: The association of Vit $\mathrm{B}_{12}$ to Gantrez AN nanoparticles was performed by the direct attachment of reactive Vit $\mathrm{B}_{12}$ to the surface of the nanoparticles (NPB), or linking to the copolymer chains in dimethylformamide prior to NP formation (NPBDMF). Nanoparticles were characterized by measuring the size, zeta potential, Vit $\mathrm{B}_{12}$ association efficacy, and stability of Vit $\mathrm{B}_{12}$ on the surface of the nanoparticles. In vivo bioadhesion study was performed by the oral administration of fluorescently-labeled nanoparticle formulations to rats. Both systemic and mucosal immune responses were evaluated after oral and subcutaneous immunization with ovalbumin (OVA) containing Vit $\mathrm{B}_{12}$-coated nanoparticles.

RESULTS: The Vit $\mathrm{B}_{12}$ nanoparticles displayed homogenous size distribution with a mean diameter of about $200 \mathrm{~nm}$ and a negative surface charge. The association efficiency of Vit $\mathrm{B}_{12}$ to NPB-DMF formulation was about two times higher than to the NPB, showing also a higher surface stability of Vit $B_{12}$. The bioadhesion study demonstrated that NPB-DMF had an important tropism to the distal portions of the gut, which was about 2 and 3.5 times higher than the tropism observed for NPB and control $\mathrm{NP}$, respectively $(\mathrm{P}<0.05)$. Oral administration of OVA-NPB-DMF induced also stronger and more balanced serum anti-OVA titers of IgG2a (Th1) and IgG1 (Th2) compared to control OVA-NP. In addition, oral immunization with OVA-NPB-DMF induced a higher mucosal $\operatorname{IgA}$ response than subcutaneous administration.

CONCLUSIONS: These results indicate the benefits of bioadhesive Vit $\mathrm{B}_{12}$-coated nanoparticles in oral antigen delivery eliciting systemic and mucosal immune response. 
Key words: Nanoparticles, Bioadhesion, Oral antigen delivery, Vitamin $\mathrm{B}_{12}$ 


\section{Introduction}

Vitamin $\mathrm{B}_{12}$ (cobalamin) has its own intestinal uptake pathway by which it can be transported to the systemic circulation. By this mechanism, a molecule of intrinsic factor (IF), secreted by the parietal cells in the stomach, interacts and binds to one molecule of free vitamin $\mathrm{B}_{12}$. The resulting complex [vitamin $\mathrm{B}_{12}-\mathrm{IF}$ ] moves down the gastrointestinal tract until it reaches the ileum. In this region, the complex is recognized by the intrinsic factor specific receptors (IF-CR) which are located on the surface of the ileal epithelial cells. Then, this complex is internalized by receptor-mediated endocytosis. Vitamin $B_{12}$ is subsequently transported to the systemic circulation and complexed with cobalamin-binding protein [Transcobalamin II (TCII)] $(1,2)$. This specific of vitamin $B_{12}$ uptake system has been explored in the last years to enhance the oral delivery of poorly available therapeutic molecules including, granulocyte colony stimulating factor, erythropoietin, and luteinizing hormone-releasing hormone (3-5). However, in spite of the numerous advances for the oral delivery of small peptides and proteins, some disadvantages have limited the efficiency of this strategy. The directly linked macromolecular drugs to vitamin $\mathrm{B}_{12}$ are susceptible to the degradation within the gastrointestinal tract. In addition, according to the limited uptake capacity of vitamin $\mathrm{B}_{12}(2.4 \mu \mathrm{g} / \mathrm{day}$ for adult) (6), the amount of the therapeutic molecule that can be delivered as conjugate is limited. An attractive solution to overcome theses problems would be the incorporation of drug in nanoparticles coated with vitamin $\mathrm{B}_{12}$. This can offer both high loading capacity of the therapeutic molecules and the protection against the aggressive gut conditions ( $\mathrm{pH}$ and enzymatic degradation).

Studies involving vitamin $\mathrm{B}_{12}$-nanoparticles conjugates have been carried out in vitro using Caco-2 cells. They have clearly demonstrated the effective cellular uptake of nanoparticles (7) or polymeric micelles containing cyclosporine A coated with vitamin 
$\mathrm{B}_{12}$ (8). Recently, in vivo results indicated the beneficial use of vitamin $\mathrm{B}_{12}$-coated dextran nanoparticles for the oral delivery of insulin (9). However, to date, no published studies have been reported on the application of nanoparticles coated with vitamin $B_{12}$ derivatives in the mucosal immunization.

Oral antigen delivery using nanoparticles is being considered as a promising strategy for oral vaccination. Orally delivered vaccines can induce high levels of mucosal immune response and, thus, protect the host against many pathogens that use the mucosal route to invade the body $(10,11)$. Unfortunately, oral immunization using conventional antigen-loaded nanoparticles does not generally elicit a sufficient systemic immune response compared to the parenteral routes, and consequently, high and multiple oral doses are required (12). This fact is related to the low capacity of conventional antigen-loaded nanoparticles to target lymphoid tissues within the gut after an oral immunization. The association between antigen-loaded nanoparticles and specific ligands (i.e., lectins) may enhance this targeting, resulting in a successful antigen delivery to the mucosal surfaces and the induction of the immune response (13, 14).

Consequently, our work strategy was to explore the beneficial use of vitamin $\mathrm{B}_{12}$ as nanoparticles' targeted ligand to achieve specific bioadhesive profiles within the gut. Thus, the main tasks were to optimize the preparation of vitamin $\mathrm{B}_{12}$-coated Gantrez AN nanoparticles, and to investigate the in vivo bioadhesive capacity after their oral administration to laboratory animals. In addition, the potential immunoadjuvant capacity of vitamin $\mathrm{B}_{12}$ nanoparticles was investigated using ovalbumin as standard antigen model. 


\section{Materials and Methods}

\section{Chemicals}

Poly(methyl vinyl ether-co-maleic anhydride) (PVM-MA) Gantrez ${ }^{\circledR}$ AN 119 was supplied by ISP (Barcelona, Spain). Reactive vitamin $\mathrm{B}_{12}-{ }^{\prime}{ }^{\prime} \mathrm{OCONH}\left[\mathrm{CH}_{2}\right]_{6}-\mathrm{NH}_{2}$ was kindly gifted by Access Pharmaceuticals Australia (Chatswood, Australia). Rhodamine B isothiocyanate (RBITC), 1,3-diaminopropane, ovalbumin (OVA, Grad V), and protease inhibitor cocktail [(4-(2 aminoethyl) benzenesulfonyl fluoride, transepoxysuccinyl-leucyl-amido (4 guanidino) butane (E-64), bestatin, leupeptin, aprotinin, and sodium EDTA] were purchased from Sigma (Madrid, Spain). Peroxidase conjugated antibodies for IgG1, IgG2a and IgA were supplied by Nordic Immunology Labs (Via Cultek S.L, Madrid, Spain). All other reagents were of an analytical grade and supplied by Merck (Darmstadt, Germany).

\section{Preparation of Vit $\mathbf{B}_{12}$-Gantrez AN nanoparticle conjugates}

The association between Gantrez AN nanoparticles and reactive vitamin $B_{12}$ was performed by two optimized methods (Figure 1, A and B).

In method A, Gantrez AN nanoparticles were prepared using a solvent displacement method (15). In brief, $100 \mathrm{mg}$ of PVM/MA copolymer were dissolved in 5 $\mathrm{mL}$ acetone, after which the nanoparticles were formed by the addition of $10 \mathrm{~mL}$ absolute ethanol under magnetic stirring. The organic polymer solution was mixed with $200 \mu \mathrm{L}$ deionized water containing $0.5 \mathrm{mg}$ reactive vitamin $\mathrm{B}_{12}$ and magnetically stirred for $1 \mathrm{~h}$ at RT. Then, $3 \mathrm{~mL}$ of deionized water were added, and the organic solvents eliminated under reduced pressure (Büchi Rotavapor R-144, Switzerland). The resulting nanoparticles were cross-linked for $5 \mathrm{~min}$ by the addition of $100 \mu \mathrm{L}$ of $1,3-$ diaminopropane (1,3-DP, 1\% w/v) and pelleted by centrifugation at 27,000 x g for 20 
min (Sigma 3K30, Germany). The supernatant was collected for quantifying the unbound vitamin $\mathrm{B}_{12}$.

For in vivo bioadhesion studies, the centrifuged nanoparticles were fluorescently labelled by incubation with $1.25 \mathrm{mg}$ RBITC for 5 minutes at room temperature under magnetic stirring after which, the suspension was pelleted again. Both RBITC- loaded and non-loaded nanoparticles were lyophilized using sucrose 5\% as cryoprotector for $48 \mathrm{~h}$ in VIRTIS GENESIS freeze drier (Virtis, New York, USA).

In method $\mathrm{B}$, Vit $\mathrm{B}_{12}$-Gantrez AN nanoparticle conjugates were prepared by the reaction between $0.25 \mathrm{mg}$ of reactive vitamin $\mathrm{B}_{12}$ and $100 \mathrm{mg}$ Gantrez AN copolymer in 12.5 mL dimethylformamide (DMF), under magnetic stirring for $48 \mathrm{~h}$ at $\mathrm{RT}$. Then, the organic solvent was removed under reduced pressure at $60{ }^{\circ} \mathrm{C}$ for $2-3 \mathrm{~h}$. The formed thin film was dissolved in $5 \mathrm{~mL}$ acetone, and the nanoparticles were formed and processed as described in method A.

\section{Characterization of vitamin $B_{12}$-Gantrez AN nanoparticle conjugates}

The particle size and zeta potential of nanoparticles were analysed by photon correlation spectroscopy (PCS) and electrophoretic laser Doppler anemometry, respectively, using a zetamaster analyser system (Malvern Instruments, Malver, UK). The morphology of the nanoparticles was observed by scanning electron microscopy in a Zeiss DSM940 digital scanning electron microscope (Oberkochen, Germany)

The amount of nanoparticles formed from the original Gantrez AN copolymer (\% yield) was determined by gravimetry (16). The amount of vitamin $\mathrm{B}_{12}$ bound to the surface of the nanoparticles was estimated from the Vit $\mathrm{B}_{12}$ concentration difference (colorimetric assay, $\lambda 540 \mathrm{~nm}$ ) measured in the supernatant before nanoparticle incubation and after the first centrifugation step. The extent of RBITC loading onto the 
nanoparticles was similarly estimated from the RBITC concentration difference measured before nanoparticle incubation and after the second centrifugation step of the nanoparticles.

\section{In vitro release of vitamin $B_{12}$ from nanoparticles}

The stability of the linked vitamin $\mathrm{B}_{12}$ to Gantrez AN nanoparticles was considered to be a critical factor for the planned in vivo study and was, therefore, used to select the appropriate nanoparticles formulation; indeed, the presence of unbound vitamin $\mathrm{B}_{12}$ can exert an auto-inhibitory effect on the binding capacity of Vit $\mathrm{B}_{12}$-Gantrez AN nanoparticles to the mucosal cells. Therefore, $10 \mathrm{mg}$ Vit $\mathrm{B}_{12}$-Gantrez AN nanoparticles were incubated in $1 \mathrm{~mL}$ simulated gastric fluid [SGF (USP XXVIII): $\mathrm{pH} 1.2$, pepsin $0.32 \% \mathrm{w} / \mathrm{v}]$ for $1 \mathrm{~h}$ at $37 \pm 1{ }^{\circ} \mathrm{C}$. Then, the nanoparticles were collected by using dialysis tubes (Vivaspin ${ }^{\circledR} 100,000$ MWCO; VIVASPIN, Hannover, Germany). The nanoparticles were then re-incubated in $1 \mathrm{~mL}$ of simulated intestinal fluid [SIF (USP XXVIII): $\mathrm{pH} 7.5$, pancreatin $1 \% \mathrm{w} / \mathrm{v}$ ] for different time periods until $24 \mathrm{~h}$ at $37 \pm 1{ }^{\circ} \mathrm{C}$. The nanoparticles were collected by dialysis, as described above, after each incubation time point. The concentration of free vitamin $\mathrm{B}_{12}$ in all of the collected dialysates was determined by colorimetry at $540 \mathrm{~nm}$.

\section{In vivo bioadhesion study}

The bioadhesion studies were carried out using a protocol described previously (16), in compliance with the regulations of the European legislation on animal experiments (86/609/EU). Briefly, an aqueous suspension containing $10 \mathrm{mg}$ nanoparticles loaded with RBITC (approximately $45 \mathrm{mg}$ particles/ $\mathrm{kg}$ body weight) was administered by the oral gavages using 16G X 3" (76.2 mm) feeding needle (Proper and Sons, inc., 
N.Y, U.S.A) to male Wistar rats fasted overnight (average weight 225 g; Harlan, Spain). The animals were sacrificed by cervical dislocation at $0.5,1,3$ and $8 \mathrm{~h}$ post administration. The abdominal cavity was opened, and the gastrointestinal tract was removed. Then, the gut was divided into six anatomical regions: stomach (Sto), intestine (I1, I2, I3 and I4) and caecum (Ce). Each mucosa segment was opened lengthwise, rinsed with PBS and digested with $3 \mathrm{M} \mathrm{NaOH}$ for $24 \mathrm{~h}$. RBITC was extracted from the digested samples by addition of $2 \mathrm{~mL}$ methanol, vortexed for $1 \mathrm{~min}$ and centrifuged at $2,000 \mathrm{x} \mathrm{g}$ for $10 \mathrm{~min}$. Aliquots $(1 \mathrm{~mL})$ of the obtained supernatants were diluted with water $(3 \mathrm{~mL})$ and assayed for RBITC content by spectrofluorimetry at $\lambda_{\mathrm{ex}} 540 \mathrm{~nm}$ and $\lambda_{\text {em }} 580 \mathrm{~nm}$ (GENios, TECAN, Austria) to estimate the fraction of adhered nanoparticles on the mucosa. Standard curves were prepared by addition of RBITC solutions in $3 \mathrm{M} \mathrm{NaOH}(0.5-10 \mu \mathrm{g} / \mathrm{mL})$ to control tissue segments following the same steps of extraction $(\mathrm{r}=0.996)$.

In order to study the kinetic parameters of bioadhesion, the total fraction of adhered nanoparticles in the whole gastrointestinal tract was plotted versus time. From these curves, the bioadhesion parameters $\mathrm{Q}_{\max }, \mathrm{AUC}_{\mathrm{adh}}, \mathrm{T}_{\max }, \mathrm{MRT}_{\mathrm{adh}}$ and $\mathrm{K}_{\mathrm{adh}}$ were estimated from 0 to $8 \mathrm{~h}$ post administration as described previously (16) and calculated using WinNonlin 1.5 software (Pharsight Corporation, USA). Q $\max$ represents the maximal adhered quantity. $\mathrm{AUC}_{\mathrm{adh}}$ is the area under the curve described for the total adhered nanoparticles versus time. $\mathrm{T}_{\max }$ was defined as the time at which maximal adhesion in gastrointestinal tract was obtained. $\mathrm{MRT}_{\mathrm{adh}}$ indicates the mean residence time of adhering nanoparticles, and $\mathrm{K}_{\mathrm{adh}}$ the elimination rate of total adhering nanoparticles. 


\section{Preparation of OVA-loaded Vit $B_{12}$-Gantrez nanoparticles}

To study the adjuvant capacity of Vit $\mathrm{B}_{12}$-Gantrez AN nanoparticles, the model antigen ovalbumin was loaded into the nanoparticle formulations. As the bioadhesion data suggested a benefit of NPB-DMF over NPB, OVA was loaded into the Vit $\mathrm{B}_{12}$-NBPDMF nanoparticles according to method B (Figure 1). Briefly, $5 \mathrm{mg}$ OVA were sonicated in $5 \mathrm{~mL}$ acetone containing $100 \mathrm{mg}$ Gantrez AN-vitamin $\mathrm{B}_{12}$ conjugate. This organic dispersoin was magnetically stirred for $1 \mathrm{~h}$ at room temperature. The copolymer was desolvated by the addition of $10 \mathrm{~mL}$ of absolute ethanol and then $3 \mathrm{~mL}$ of deionized water. The organic solvents were eliminated under reduced pressure (Büchi R-144, Switzerland), and the resulting aqueous nanosuspension was cross-linked with 1,3-DP and pelleted by centrifugation at $27,000 \mathrm{x} \mathrm{g}$ for $20 \mathrm{~min}$. The supernatant was used to quantify the unbounded vitamin $B_{12}$. Finally, OVA-NPB-DMF nanoparticle dispersion was lyophilized, as described above. Control nanoparticles (OVA-NP) were prepared by the same way, but without the use of vitamin $\mathrm{B}_{12}$.

\section{Characterization of OVA-loaded Vit $B_{12}$-nanoparticles}

The size and zeta potential of the nanoparticles as well as the amount of vitamin $B_{12}$ associated to OVA-NPB-DMF were determined as described above. The amount of OVA associated to the OVA-NPB-DMF nanoparticles was determined by SDS-PAGE. Typically, 5 mg OVA-NPB-DMF nanoparticles were dissolved in $2 \mathrm{~mL}$ DMF/acetone $(1: 1, \mathrm{v} / \mathrm{v})$ and then kept at $-20{ }^{\circ} \mathrm{C}$ for $24 \mathrm{~h}$. Samples were centrifuged at $10,000 \mathrm{x} \mathrm{g}$ for $15 \mathrm{~min}$, and the precipitate was washed with cold acetone and centrifuged again. The amount of OVA was estimated from the average band density of the samples in the

SDS-PAGE using Micro Image ${ }^{\circledR}$ software (Version 4.0; Olympus Optical, Hamburg, Germany); an OVA standard curve was established in the range of 2.5-0.25 $\mu \mathrm{g} / \mathrm{band}$. 


\section{Immunization protocol}

Animal study protocols were applied in compliance with the regulations of the responsible committee of the University of Navarra in line with the European legislation on animal experiments (86/609/EU). Female BALB/c mice of average weight of $20 \pm$ 1g, supplied by Harlan (Barcelona, Spain), were divided into 6 groups of 10 mice. Mice were fasted overnight, with free access to water. The formulations were administered as single OVA doses by either oral (100 $\mu$ g OVA) or subcutaneous (20 $\mu$ g OVA) route. In the oral groups (3 groups, $n=10$ ), animals were fed by oral gavage of $200 \mu \mathrm{L}$ PBS containing OVA-NPB-DMF nanoparticles or OVA-NP (control nanoparticles), or OVA in solution. In the subcutaneous groups ( 3 groups, $n=10$ ), the animals were injected with $50 \mu \mathrm{L}$ PBS containing OVA-NPB-DMF nanoparticles, OVA-NP, or OVA in solution. After $2 \mathrm{~h}$ post administration, the animals were allowed unrestricted access to water and food.

\section{Sample collection}

Blood samples were collected from the retroorbital plexus on $0,1,2,4$ and 6 weeks post administration. The samples were centrifuged (10,000 x g, $10 \mathrm{~min})$, the sera pooled for each group (10 mice), diluted 1:10 with PBS and conserved at $-80{ }^{\circ} \mathrm{C}$ untill analysis. Faecal samples were collected and analysed as described previously (17). Pooled fresh pelleted faeces from each group of mice were put into microtubes on $0,1,2,4,6$ weeks post administration and weighed. Non-fatty milk (3\%) in phosphate-buffered saline was added at $1 \mathrm{~mL} / 100 \mathrm{mg}$ faecal pellets. The pellets were vortexed for $5 \mathrm{~min}$ at room temperature. Then, the tubes were centrifuged at $10,000 \times \mathrm{g}$ for $10 \mathrm{~min}$, and the supernatants transferred into tubes containing $10 \mu \mathrm{L}$ of protease inhibitor cocktail. Finally, pooled samples were storedd at $-80{ }^{\circ} \mathrm{C}$ until analysis. 


\section{ELISA assay}

\section{Serum anti-OVA IgG1 and IgG2a}

Anti-OVA serum antibodies (IgG1 and $\mathrm{IgG} 2 \mathrm{a}$ ) were assayed by ELISA using 96 microtiter plates (Thermo LabSystems, Vantaa, Finland). Wells were coated with OVA (100 $\mu \mathrm{L}$ per well) in a solution of $10 \mu \mathrm{g} / \mathrm{mL}$ of OVA in $0.05 \mathrm{M}$ sodium carbonatebicarbonate buffer ( $\mathrm{pH}$ 9.6) at $4{ }^{\circ} \mathrm{C}$ for $24 \mathrm{~h}$. Plates were washed 3 times with PBS containing $0.05 \%$ Tween 20 (PBS-T) using an ELISA plate washer (Thermo Labsystems, Vantaa, Finlandia). Then, wells were incubated with $200 \mu \mathrm{L}$ of $1 \%$ BSA in PBS-T for $1 \mathrm{~h}$ at room temperature. After washing as described above, serum samples $(100 \mu \mathrm{L})$ were added in twofold serial dilutions in PBS-T starting with 1:40 initial dilution, and incubated at $37^{\circ} \mathrm{C}$ for $4 \mathrm{~h}$. The washed wells were incubated for $2 \mathrm{~h}$ at 37 ${ }^{\circ} \mathrm{C}$ with $100 \mu \mathrm{L}$ of anti-IgG $\operatorname{Ig}_{1}$ and anti- $\operatorname{IgG}_{2 \mathrm{a}}$ peroxidase-conjugated antibodies (GAM/IgG1/PO and GAM/IgG2a/PO) used in dilution of 1:1000 in PBS-T. For color development, the substrate-chromogen used was $\mathrm{H}_{2} \mathrm{O}_{2}$-ABTS (3-ethylbenzthiazoline-6sulfonic acid). The absorbance (Abs) was determined at $405 \mathrm{~nm}$ (iEMS Reader MF de Labsystems, Vantaa, Finlandia), and the end-point titer determined as the sample dilution giving a mean $\mathrm{Abs} \geq 0.2$ above that measured for untreated mice sera.

For semi-quantitative comparison between immune responses induced by the different nanoparticle formulations, the areas under the curves of the antibody response profiles $\left(\mathrm{AUC}_{\mathrm{th} 1(\mathrm{IgG} 2 \mathrm{a})}\right.$ and $\left.\mathrm{AUC}_{\mathrm{th} 2(\mathrm{IgG1})}\right)$ were calculated for the period of 0 - 6 weeks post immunization using the WinNonline 1.5 software (Pharsight Corp, USA), as described previously (16). 


\section{Faecal anti-OVA IgA}

After the washing step of OVA-coated plates, as described above, the plates were blocked with $200 \mu \mathrm{L} 3 \%$ non-fatty milk in PBS-T for $1 \mathrm{~h}$ at room temperature. Faecal extract samples of $100 \mu \mathrm{L}$ were added starting with undiluted samples and followed by twofold serial dilutions up to 1:64 in PBS-T; the plates were incubated at $37^{\circ} \mathrm{C}$ for $4 \mathrm{~h}$. After washing the wells, 1:1000 dilutions of anti-IgA peroxidase conjugated antibody (GAM/IgA/PO) were added. The detection step and the end titers were determined as described above.

\section{Statistical methods}

The bioadhesion data and the physico-chemical characteristics were compared using the nonparametric Mann-Whitney U-test and Student t-test, respectively. P values of $<0.05$ were considered significant. All calculations were performed using SPSS ${ }^{\circledR}$ statistical software program $\left(\operatorname{SPSS}^{\circledR} 10\right.$, Microsoft, USA). 


\section{Results}

\section{Characteristics of nanoparticle formulations}

Vit $\mathrm{B}_{12}$-nanoparticle conjugates were prepared by two different methods. In method A, vitamin $B_{12}$ was bound to the surface of previously prepared nanoparticles. In method $\mathrm{B}$, a Vit $\mathrm{B}_{12}$-Gantrez AN conjugate was first prepared, which was subsequently used to prepare the nanoparticles. All nanoparticle formulations were prepared with similar yields (about 80\%) and showed homogenous size distributions with a mean diameter of about $200 \mathrm{~nm}$ (Table 1, Figure 2). Both types of Vit $\mathrm{B}_{12}$-nanoparticles (NPB and NPBDMF; see Figure 1) displayed a similar negative zeta potential, which differed significantly from that measured with control nanoparticles $(\mathrm{P}<0.05)$ (Table 1$)$. The content of vitamin $\mathrm{B}_{12}$ was higher in the NPB-DMF nanoparticles than in the NPB nanoparticles $(\mathrm{P}<0.05)$. In addition, the association efficiency of vitamin $\mathrm{B}_{12}$ to the nanoparticles prepared by method B was two times higher than that obtained with the nanoparticles prepared by method A (Table 1). Finally, the amount of loaded RBITC was similar, i.e., appro.x $13 \mu \mathrm{g} / \mathrm{mg}$ nanoparticles, for all the nanoparticle formulations.

\section{In vitro release of vitamin $B_{12}$ from the nanoparticles}

Figure 3 illustrates the release of vitamin $B_{12}$ from nanoparticles after their subsequent incubation in simulated gastric fluid (SGF) and intestinal fluid (SIF). After $1 \mathrm{~h}$ incubation in SGF ( $\mathrm{pH}$ 1.2), NPB produced a significant release of vitamin $\mathrm{B}_{12}$, which was about $30 \%$ of the total amount of associated vitamin $\mathrm{B}_{12}$. When this formulation was subsequently incubated in SIF ( $\mathrm{pH} 7.5$ ), the release of vitamin $\mathrm{B}_{12}$ continued in a more sustained way and reached approx. 50\% of the total dose within $24 \mathrm{~h}$. On the contrary, less than $3 \%$ of vitamin $\mathrm{B}_{12}$ were released from NPB-DMF after their incubation in SGF (1 h) and SIF (23 h). 


\section{In vivo bioadhesion study}

Figure 4 shows the distribution of the adhered nanoparticles throughout the different sections of the GI tract after a single oral administration of $10 \mathrm{mg}$ RBITC-loaded nanoparticles. Thirty minutes after administration, the Vit $\mathrm{B}_{12}$-nanoparticles (NPB, NPB-DMF) and control nanoparticles (NP) displayed a similar capacity to adhere to the gut, with a preferential distribution in the stomach and upper regions of the small intestine (Figure 4 a,b,c). Interestingly, at $3 \mathrm{~h}$ post administration, both Vit $\mathrm{B}_{12^{-}}$ nanoparticle formulations displayed a higher tropism for the distal part of the gut (I3 \& I4 portions) than the control nanoparticles (NP). Furthermore, this tropism was 2 and 3.5-times higher with the NPB-DMF nanoparticles than with the NPB and control nanoparticles (NP), respectively.

Analysis of the bioadhesion kinetics (Figure 5) revealed that control nanoparticles achieved their maximal gut adhesion within the first $30 \mathrm{~min}$, whereas the Vit $\mathrm{B}_{12^{-}}$ nanoparticle formulations required $3 \mathrm{~h}$ to reach $\mathrm{T}_{\max }$ (Table 2). On the other hand, both the maximum amount $\left(\mathrm{Q}_{\max }\right)$ and total amount $\left(\mathrm{AUC}_{\mathrm{adh}}\right)$ of adhered nanoparticles were significantly higher for the NPB-DMF nanoparticles than for the NPB or control nanoparticles $(\mathrm{p}<0.01)$. The mean residence time of the adhered nanoparticle fraction $\left(\mathrm{MTR}_{\mathrm{adh}}\right)$ was similar, i.e., approx. $3.5 \mathrm{~h}$, for all nanoparticle formulations. Finally, the elimination constant $\left(\mathrm{K}_{\mathrm{adh}}\right)$ for the adhered fraction of nanoparticles was significantly higher for the NPB-DMF nanoparticles than for the other formulations (Table 2).

\section{Characteristics of OVA-loaded Vit $B_{12}$ nanoparticles}

For the immunization studies, the model antigen OVA was encapsulated in the Vit $\mathrm{B}_{12^{-}}$ nanoparticle formulation NPB-DMF and control nanoparticles free of vitamin $\mathrm{B}_{12}(\mathrm{NP})$. 
The OVA-NPB-DMF nanoparticles displayed a slightly lower mean diameter $(230 \mathrm{~nm})$ than the control OVA-loaded nanoparticles (OVA-NP, $270 \mathrm{~nm}$ ). The amount of vitamin $\mathrm{B}_{12}$ associated to OVA-NPB-DMF nanoparticles was approx. $2.4 \mu \mathrm{g} / \mathrm{mg}$ NP. The amount of OVA loaded in the nanoparticles was approx. $12 \mu \mathrm{g}$ OVA/mg NP and similar for both the OVA-NPB-DMF nanoparticles and OVA-NP.

\section{OVA-specific serum antibody response}

Figure 6 shows OVA-specific IgG2a and IgG1 serum titers measured after oral gavage or subcutaneous (s.c.) administration of a single dose of OVA-loaded Vit $\mathrm{B}_{12^{-}}$ nanoparticles (OVA-NPB-DMF), OVA-loaded control nanoparticles free of vitamin $\mathrm{B}_{12}$ (OVA-NP) and free OVA. After both the oral and s.c. administrations, both nanoparticle formulations enhanced the serum antibody responses (IgG2a, IgG1) compared to free antigen. Upon s.c. immunization, the $\operatorname{IgG} 2 \mathrm{a}$ titers (mirroring a Th1 response) elicited by the OVA-NPB-DMF nanoparticles were at least two times higher than those elicited by the OVA-NP (see AUC-values, Figure 6 a,b), although both OVA-loaded nanoparticle formulations (OVA-NPB-DMF and OVA-NP) induced similar IgG1 titers (mirroring a Th2 response).

Oral immunization with the control OVA-NP showed a predominant IgG1 response $\left(\mathrm{AUC}_{\mathrm{IgG} 1}\right.$ was seven times higher than $\mathrm{AUC}_{\mathrm{IgG} 2 \mathrm{a}}$, Figure $\left.6 \mathrm{c}, \mathrm{d}\right)$. In contrast, oral immunization with OVA-NPB-DMF elicited stronger and more balanced Th1/Th2 responses as compared to the control OVA-NP. The sum of both the $\mathrm{AUC}_{\mathrm{IgG} 1}$ and $\mathrm{AUC}_{\mathrm{IgG} 2 \mathrm{a}}$ for OVA-NPB-DMF was about three times higher than that calculated for OVA-NP (Figure $6 \mathrm{c}, \mathrm{d}$ ). 


\section{Anti-OVA specific intestinal IgA}

Figure 7 illustrates the faecal anti-OVA $\operatorname{IgA}$ response after subcutaneous and oral immunization of the different formulations tested. Both nanoparticle formulations elicited higher levels of intestinal IgA compared to free OVA when administered by either the s.c. or oral route. Interestingly, the oral administration of OVA-NPB-DMF elicited higher levels of intestinal secretory IgA compared to the s.c. administration. This mucosal immune response (IgA) was about four $\log _{2}$-titers (approx. 100-fold) higher than that elicited by OVA-NP. 


\section{Discussion}

The first objective of this work was to optimize the preparation of Vit $\mathrm{B}_{12}$-Gantrez AN nanoparticle conjugates. For this purpose, the association of vitamin $B_{12}$ to the nanoparticles was done by applying two different methods, where vitamin $\mathrm{B}_{12}$ was either directly attached to the surface of the nanoparticles (NPB, method A), or linked to the copolymer chains prior to nanoparticle formation (NPB-DMF, method B). Both Vit $\mathrm{B}_{12^{-}}$nanoparticle types displayed a homogenous size distribution with mean diameter of about $200 \mathrm{~nm}$, and a less negative surface charge compared to control nanoparticles (NP). The high density of negative surface charge of the control NP is due to the exposure of hydrolyzed carboxylic groups of Gantrez AN copolymer. The attachment of primary amine-containing molecules such as 1,3-diaminopropane, lectins or proteins to the surface of Gantrez nanoparticles decreases their negative surface charge $(15,18)$. This may explain the significant decrease in the negative surface charge of both types of vitamin $\mathrm{B}_{12}$ nanoparticles when reactive vitamin $\mathrm{B}_{12}-\mathrm{NH}_{2}$ was directly attached to Gantrez nanoparticles or linked to the copolymer.

During the optimization of the nanoparticle preparation methods (A and B) we noticed that method B (NPB-DMF; $250 \mu \mathrm{g}$ vitamin $\mathrm{B}_{12}$ per $100 \mathrm{mg}$ Gantrez $\mathrm{AN}$ in DMF) required only half of the amount of reactive vitamin $\mathrm{B}_{12}$ to yield a loading of approx. $2.4 \mu \mathrm{g} / \mathrm{mg}$ NP (Table 1) than method A (NPB; $500 \mu \mathrm{g}$ vitamin $\mathrm{B}_{12}$ per $100 \mathrm{mg}$ Gantrez AN nanoparticles, in an aqueous dispersion). This result correlated with the twofold higher association efficiency of vitamin $\mathrm{B}_{12}$ to the NPB-DMF as compared to the NPB nanoparticles. 
The physical stability of association of vitamin $\mathrm{B}_{12}$ and Gantrez AN nanoparticles was considered as a critical factor for the in vivo experiments, since free vitamin $\mathrm{B}_{12}$ was expected to exert an auto-inhibitory effect on the mucosal affinity of Vit $\mathrm{B}_{12}$-Gantrez AN nanoparticles after their oral administration. Therefore, the release of vitamin $B_{12}$ was studied upon subsequent incubation of the nanoparticles in simulated gut fluids. The release of vitamin $B_{12}$ form both types of nanoparticles showed that the vitamin $B_{12}$ remained more strongly associated to NPB-DMF than to the NPB nanoparticles (Figure $3)$.

The differences in vitamin $B_{12}$ association efficiency stability between the two nanoparticle formulations may depend on the reaction conditions under which vitamin $\mathrm{B}_{12}$ was coupled to Gantrez AN copolymer (NPB-DMF) or linked to the surface of the nanoparticles (NPB). The expected formation of stable amide bonds between the primary amine group of vitamin $\mathrm{B}_{12}-5^{\prime} \mathrm{OCONH}\left[\mathrm{CH}_{2}\right]_{6}-\mathrm{NH}_{2}$ and the anhydride group of Gantrez AN nanoparticles (19) may have been hampered in the presence of water used for this reaction with the NPB. Indeed, the high hydrolysis rate of the anhydride groups of the poly(methyl vinyl ether-co-maleic anhydride) may have produced carboxylic groups that formed less stable charged ionic or hydrogen bonds with the reactive vitamin $\mathrm{B}_{12}$ derivative. Conversely, the coupling of vitamin $\mathrm{B}_{12}$ to the copolymer (NPBDMF) in the absence of water must have yielded a high fraction of stable covalent bonds between vitamin $\mathrm{B}_{12}$ and the copolymer. These observations are in agreement with previous results obtained for the pegylation of Gantrez AN nanoparticles (20), where a threefold higher pegylation of Gantrez nanoparticles was achieved when polyethylene glycol was reacted with Gantrez in an organic phase prior to nanoparticle 
formation as compared to the coating of freshly prepared nanoparticles with polyethylene glycol.

The in vivo bioadhesive capacity of Vit $\mathrm{B}_{12}$-nanoparticles was investigated after oral administration of fluorescently labelled NPB and NPB-DMF to fasted rats. The adhered fractions of nanoparticles were mainly found in the stomach and upper regions of the small intestine at 30 min post administration (Figure 4 a,b,c). At 3 hours post administration, both $\mathrm{Vit} \mathrm{B}_{12}$-nanoparticle formulations showed an important tropism to the distal portions of the gut (I3 \& I4 regions). The tropism of the NPB-DMF nanoparticles represented about $28 \%$ of the given dose and was about 2 and 3.5 times more than the tropism observed for the NPB and control nanoparticles, respectively $(\mathrm{P}<$ 0.05). From a physiological point of view, the ileum is the region of the gastrointestinal tract where the Vit $\mathrm{B}_{12}$-intrinsic factor (IF) complex is transported (1). Therefore, these results appear to demonstrate the biological property of vitamin $\mathrm{B}_{12}$ on the surface of the nanoparticles (NPB-DMF) to bind effectively to the IF, with subsequent binding of the IF-Vit $\mathrm{B}_{12}$ - nanoparticles to the IF receptors of the mucosal cells.

Interestingly, we noted a good agreement between the vitamin $\mathrm{B}_{12}$ release (Figure 3) and the bioadhesive capacity (ileal tropism) of the two types of vitamin $\mathrm{B}_{12}$ nanoparticles (Figure 4). The twofold enhanced ileal tropism of NPB-DMF nanoparticles over that of the NPB may be related to the strong association of vitamin $\mathrm{B}_{12}$ to the NPB-DMF nanoparticles (Figure 3), whereas the important release of vitamin $\mathrm{B}_{12}$ from the NPB (Figure 3) probably exerted an auto-inhibitory effect on the ileal tropism of the nanoparticles. Such competing effect between orally administered free vitamin $\mathrm{B}_{12}$ and vitamin $\mathrm{B}_{12}$-peptide conjugates, which negatively affected the process 
of uptake and transport to the circulation of the conjugate, has been described in the literature (21).

NPB-DMF and NPB nanoparticles reached the maximal gut adhesion at $3 \mathrm{~h}$ post administration (Figures 4 and 5, Table 2), whereas the control nanoparticles achieved their maximal bioadhesion already at $0.5 \mathrm{~h}$ post administration. These results indicate that control nanoparticles without vitamin $\mathrm{B}_{12}$ on their surface distribute quickly in the stomach and upper ileal segments, which is mediated by non-specific bioadhesive interactions between the Gantrez AN copolymer and the mucosal surfaces. However, the presence of vitamin $B_{12}$ on the surface of the nanoparticles prolongs substantially the time needed to reach the maximal gut adhesion, which we ascribe to specific bioadhesive interactions between the vitamin $\mathrm{B}_{12}$ and the IF-receptor of the mucosal cells.

Arbós and co-workers demonstrated that surface modifications of conventional Gantrez AN nanoparticles with lectins can delay the time of maximal bioadhesion of control nanoparticles from $0.5 \mathrm{~h}$ to $1 \mathrm{~h}$ post oral administration (16). Similarly, oral administration of vitamin $\mathrm{B}_{12}$-conjugates with peptides to rats yielded the highest tissue concentration in the small intestine after 4 hours post oral administration (21). The time of 4 hours agrees well with our data suggesting that similar bioadhesive kinetics determined the fate of both the vitamin $\mathrm{B}_{12}$-peptide and vitamin $\mathrm{B}_{12}$-nanoparticle conjugates in the same animal model.

Finally, to investigate the adjuvant capacity of Vit $\mathrm{B}_{12}$-nanoparticles as antigen delivery system, the antibody immune response was studied in BALB/c mice. Both s.c. and oral 
administration of the OVA-loaded nanoparticle formulations elicited a stronger serum antibody response than free OVA. In the s.c. immunization, the OVA-NP induced a considerable IgG1 (Th2) response, whereas the OVA-loaded Vit $\mathrm{B}_{12}$-nanoparticles (OVA-NPB-DMF) induced higher IgG2a levels (Th1 response) than OVA-NP. The IgG2a response may be related to effective uptake of Vit $\mathrm{B}_{12}$-nanoparticles by antigen presenting cells, although this hypothesis requires further investigation. The intragastric inoculation of the OVA-NPB-DMF formulation induced also stronger and more balanced serum titers of IgG2a (Th1) and IgG1 (Th2) compared to the OVA-NP, which induced a typical Th2 response (Figure $6 \mathrm{c}, \mathrm{d}$ ). For semi-quantitative analysis, the cumulated areas under the curve of the antibody response profiles $\left(\mathrm{AUC}_{\mathrm{IgG2a}}+\right.$ $\left.\mathrm{AUC}_{\mathrm{IgG} 1}\right)$ were found to be approx. three times larger for OVA-NP-DMF than for OVANP. This emphasized again the strong adjuvant capacity of Vit $\mathrm{B}_{12}$-nanoparticles when loaded with ovalbumin.

These results clearly indicated the capacity of Vit $\mathrm{B}_{12}$-nanoparticles to enhance a Th1 response after both s.c. and oral immunization of BALB/c mice, which have a genetic tendency towards a Th2 response (22). This Th1 response enhancement may be related to the high ileal tropism of Vit $\mathrm{B}_{12}$-nanoparticles, where Peyer's patches with a high density of antigen presenting cells are found to be concentrated. In contrast, control nanoparticles did not show ileal tropism and elicited only a week Th2 type immune response. These results agree with those reported on the delivery of OVA-loaded microbeads to lower intestine region, where they enhanced a Th1 response (23). Finally, the oral immunization with OVA-NPB-DMF induced a higher mucosal IgA response than the s.c. administration. In summary, these results support further studies in order to 
apply Vit $\mathrm{B}_{12}$-Gantrez $\mathrm{AN}$ nanoparticles in protective mucosal vaccination or immunotherapy purposes.

\section{Conclusions}

The present results demonstrate the adjuvant benefits of vitamin $\mathrm{B}_{12}$ association to Gantrez AN nanoparticles for oral antigen delivery. The bioadhesive capacity and ileal tropism of the orally administered OVA-loaded and vitamin $\mathrm{B}_{12}$-decorated nanoparticles appeared to be instrumental for the effective elicitation of both systemic and mucosal immune responses. Further efforts should be focused on exploring the role of vitamin $B_{12}$ in the interaction with immune cells and their potential activation.

\section{Acknowledgements}

This work was supported by grants from the "Ministerio de Educación y Ciencia de España, CICYT" (Projects SAF 2001-0690-C03 and AGL2004-07088-CO3-02/GAN), “Agencia Española de Cooperacion Internacional, AECI", Foundations "Universitaria de Navarra" and "María Francisca de Roviralta", and "Asociación de Amigos Universidad de Navarra" in Spain. 


\section{References}

1. G. J. Russell-Jonesand D. H. Alpers. Vitamin B12 transporters. Pharm Biotechnol 12: 493-520 (1999).

2. C. H. Halsted. Absorption of water-soluble vitamins. Curr Opin Gastroenterol 19: 113-7 (2003).

3. A. Habberfield, K. Jenson-Pippo, L. Ralph, S. W. Westwood, and G. J. RussellJones. Vitamin B12-mediated uptake of erythropoietin and granulocyte colony stimulating factor in vitro and in vivo. Int. J. Pharm. 145: 1-8 (1996).

4. G. J. Russell-Jones, S. W. Westwood, and A. D. Habberfield. Vitamin B12 mediated oral delivery systems for granulocyte-colony stimulating factor and erythropoietin. Bioconjug Chem 6: 459-65 (1995).

5. G. J. Russell-Jones, S. W. Westwood, P. G. Farnworth, J. K. Findlay, and H. G. Burger. Synthesis of LHRH antagonists suitable for oral administration via the vitamin B12 uptake system. Bioconjug Chem 6: 34-42 (1995).

6. M. V. Bor, E. Lydeking-Olsen, J. Moller, and E. Nexo. A daily intake of approximately 6 microg vitamin B-12 appears to saturate all the vitamin B-12related variables in Danish postmenopausal women. Am J Clin Nutr 83: 52-8 (2006).

7. G. J. Russell-Jones, L. Arthur, and H. Walker. Vitamin B12-mediated transport of nanoparticles across Caco-2 cells. Int J Pharm 179: 247-55 (1999).

8. M. F. Francis, M. Cristea, and F. M. Winnik. Exploiting the vitamin B12 pathway to enhance oral drug delivery via polymeric micelles. Biomacromolecules 6: 2462-7 (2005).

9. K. B. Chalasani, G. J. Russell-Jones, S. K. Yandrapu, P. V. Diwan, and S. K. Jain. A novel vitamin B12-nanosphere conjugate carrier system for peroral delivery of insulin. J Control Release 117: 421-9 (2007).

10. W. S. Shalaby. Development of oral vaccines to stimulate mucosal and systemic immunity: barriers and novel strategies. Clin Immunol Immunopathol 74: 127-34 (1995).

11. J. R. McGheeand J. Mestecky. In defense of mucosal surfaces. Development of novel vaccines for $\operatorname{IgA}$ responses protective at the portals of entry of microbial pathogens. Infect Dis Clin North Am 4: 315-41 (1990).

12. M. Hori, H. Onishi, and Y. Machida. Evaluation of Eudragit-coated chitosan microparticles as an oral immune delivery system. Int J Pharm 297: 223-34 (2005).

13. F. Walter, I. Scholl, E. Untersmayr, A. Ellinger, G. Boltz-Nitulescu, O. Scheiner, F. Gabor, and E. Jensen-Jarolim. Functionalisation of allergen-loaded microspheres with wheat germ agglutinin for targeting enterocytes. Biochem Biophys Res Commun 315: 281-7 (2004).

14. E. C. Lavelle, G. Grant, U. Pfuller, and D. T. O'Hagan. Immunological implications of the use of plant lectins for drug and vaccine targeting to the gastrointestinal tract. J Drug Target 12: 89-95 (2004). 
15. P. Arbos, M. Wirth, M. A. Arangoa, F. Gabor, and J. M. Irache. Gantrez AN as a new polymer for the preparation of ligand-nanoparticle conjugates. $J$ Control Release 83: 321-30 (2002).

16. P. Arbos, M. A. Arangoa, M. A. Campanero, and J. M. Irache. Quantification of the bioadhesive properties of protein-coated PVM/MA nanoparticles. Int $J$ Pharm 242: 129-36 (2002).

17. M. Maciel, A. E. Fusaro, C. R. Oliveira, E. A. Futata, A. J. Duarte, and M. N. Sato. IgA response in serum and gut secretion in sensitized mice fed with the dust mite Dermatophagoides pteronyssinus extract. Braz J Med Biol Res 37: 817-26 (2004).

18. P. Arbos, M. A. Campanero, M. A. Arangoa, M. J. Renedo, and J. M. Irache. Influence of the surface characteristics of PVM/MA nanoparticles on their bioadhesive properties. J Control Release 89: 19-30 (2003).

19. U. Schmidt, S. Zschoche, and C. Werner. Modification of poly (octadecene-altmaleic anhydride) films by reaction with functional amines. J. Appl. Polym. Sci. 87: 1255 (2002).

20. K. Yoncheva, E. Lizarraga, and J. M. Irache. Pegylated nanoparticles based on poly(methyl vinyl ether-co-maleic anhydride): preparation and evaluation of their bioadhesive properties. Eur J Pharm Sci 24: 411-9 (2005).

21. J. Alsenz, G. J. Russell-Jones, S. Westwood, B. Levet-Trafit, and P. C. de Smidt. Oral absorption of peptides through the cobalamin (vitamin B12) pathway in the rat intestine. Pharm Res 17: 825-32 (2000).

22. R. S. Gieni, Y. Fang, G. Trinchieri, D. T. Umetsu, and R. H. DeKruyff. Differential production of IL-12 in BALB/c and DBA/2 mice controls IL-4 versus IFN-gamma synthesis in primed CD4 lymphocytes. Int Immunol 8: 151120 (1996).

23. R. I. Cronkhiteand J. G. Michael. Sub-compartmentalization of the gastrointestinal (GI) immune system determined with microbeads that differ in release properties. Vaccine 22: 2106-15 (2004). 
Table 1. Physicochemical characteristics of the Vit $\mathrm{B}_{12}$-nanoparticles. Data expressed as mean $\pm \operatorname{SD}(n=6)$.

\begin{tabular}{|c|c|c|c|c|c|c|}
\hline & $\begin{array}{c}\text { Size } \\
(\mathbf{n m})^{\mathrm{a}}\end{array}$ & PDI $^{b}$ & $\begin{array}{c}\text { Zeta } \\
\text { potential } \\
(\mathrm{mV})^{\mathbf{c}}\end{array}$ & $\begin{array}{c}\text { Yield }^{\mathrm{d}} \\
(\%)\end{array}$ & $\begin{array}{c}\text { Vit } B_{12} \\
\text { content } \\
(\mu \mathrm{g} / \mathrm{mg})^{\mathrm{e}}\end{array}$ & $\begin{array}{c}\text { Association } \\
\text { efficiency } \\
\text { of vitamin } B_{12} \\
\text { to } \mathrm{NP}(\%)^{\mathbf{f}} \\
\end{array}$ \\
\hline NP & $203 \pm 1$ & $0.15 \pm 0.04$ & $-47 \pm 1$ & $78 \pm 1$ & - & - \\
\hline NPB & $200 \pm 3$ & $0.16 \pm 0.03$ & $-24 \pm 3^{*}$ & $83 \pm 1$ & $2.32 \pm 0.07$ & $38 \pm 1$ \\
\hline NPB-DMF & $198 \pm 1$ & $0.09 \pm 0.08$ & $-26 \pm 2 *$ & $79 \pm 2$ & $2.45 \pm 0.05$ & $77 \pm 1 *$ \\
\hline
\end{tabular}

NPB and NPB-DMF: Vit $B_{12}$-nanoparticles prepared by methods A and B (see Figure 1)

${ }^{a}$ Measurement of the nanoparticle size by photon correlation spectroscopy

${ }^{\mathrm{b}}$ Polydispersity index

${ }^{\mathrm{c}}$ Measurement of the zeta potential by electrophoretic laser Doppler anemometry

${ }^{\mathrm{d}}$ Percentage of nanoparticles formed relative to the initial amount of polymer used

${ }^{\mathrm{e}}$ Amount of vitamin $\mathrm{B}_{12}$ associated to the nanoparticles expressed in $\mu \mathrm{g} / \mathrm{mg} \mathrm{NP}$

${ }^{\mathrm{f}}$ Association efficiency of vitamin $\mathrm{B}_{12}$ to the nanoparticles formulation expressed in percentage relative to the initial amounts of materials used

$* \mathrm{P}<0.05 ;$ MNPB and NPB-DMF versus control nanoparticles (NP) (Student t-test). 
Table 2. Bioadhesion kinetic parameters for the tested formulations. Data expressed as

$$
\text { mean } \pm \operatorname{SD}(\mathrm{n}=3)
$$

\begin{tabular}{|c|c|c|c|c|c|}
\hline & $\mathbf{Q}_{\max }(\mathbf{m g})$ & $\mathbf{T}_{\max }(\mathbf{h})$ & $\mathbf{A U C}_{\mathbf{a d h}}(\mathbf{m g . h})$ & $\mathbf{K}_{\mathbf{a d h}}\left(\mathbf{h}^{\mathbf{- 1}}\right)$ & $\mathbf{M R T}_{\text {adh }}(\mathbf{h})$ \\
\hline $\mathbf{N P}$ & $2.13 \pm 0.18$ & 0.5 & $10.95 \pm 0.1$ & $0.13 \pm 0.08$ & $3.45 \pm 0.32$ \\
\hline NPB & $2.17 \pm 0.46$ & 3 & $12.87 \pm 2.1$ & $0.11 \pm 0.01$ & $3.77 \pm 0.14$ \\
\hline NPB-DMF & $3.61 \pm 0.73^{* *}$ & 3 & $24.41 \pm 2.2^{* *}$ & $0.22 \pm 0.08^{*}$ & $3.53 \pm 0.24$ \\
\hline
\end{tabular}

NPB and NPB-DMF: Vit $B_{12}$-nanoparticles prepared by methods A and B (see figure 1).

$\mathbf{Q}_{\max }(\mathbf{m g})$ : Maximal amount of nanoparticles adhering to the intestinal mucosa

$\mathbf{A U C}_{\mathbf{a d h}}(\mathbf{m g} \cdot \mathbf{h})$ : Area under the curve of nanoparticles adhering to the intestinal mucosa

$\mathbf{K}_{\text {adh }}\left(\mathbf{h}^{-1}\right)$ : Terminal elimination rate of the adhered fraction of nanoparticles

$\mathbf{M R T}_{\text {adh }}(\mathbf{h})$ : Mean residence time of the adhered fraction of nanoparticles

$\mathbf{T}_{\text {max }}$ : The time needed to reach the maximal adhesion

$* P<0.05, * * P<0.01$ for NPB-DMF versus control nanoparticles NP (Man-Whitney U-test). 


\section{Legend to figures}

Figure 1. Schematic representation of the methods A and B used for preparing the Gantrez AN nanoparticles and associating vitamin $B_{12}$ to the nanoparticles.

Figure 2. Scanning electron micrographs $(\mathrm{SEM})$ of lyophilized Vit $\mathrm{B}_{12}$-nanoparticles (NPB-DMF).

Figure 3. Vitamin $B_{12}$ release profiles from Vit $B_{12}$-Gantrez nanoparticles upon their subsequent incubation in simulated gastric and intestinal fluids. The nanoparticle formulations were: $(\bullet)$ NPB and $(\bullet)$ NPB-DMF. Data represented as mean \pm SD $(n=3)$.

Figure 4. Distribution of nanoparticles in the gastrointestinal tract of rats after oral administration of $10 \mathrm{mg}$ RBITC-loaded nanoparticles. (a) Control nanoparticles (NP); (b) Vit $\mathrm{B}_{12}-\mathrm{NP}$ prepared by method $\mathrm{A}(\mathrm{NPB})$; (c) Vit $\mathrm{B}_{12}-\mathrm{NP}$ prepared by method $\mathrm{B}$ (NPB-DMF). The $\mathrm{x}$-axis represents the different gut segments of stomach (Sto), intestinal sections (I1, I2, I3, I4), and caecum (Ce). The y-axis shows the adhered amounts of nanoparticles on the mucosa. The $\mathrm{z}$-axis represents the post administration time. Each value is represented by the mean ( $n=3$; SD was less than $20 \%$ of the mean).

Figure 5. Kinetics of bioadhesion of Vit $\mathrm{B}_{12}$-nanoparticles throught the whole gastrointestinal tract versus time: $(\bullet) \mathrm{NPB},(\boldsymbol{\bullet}) \mathrm{NPB}-\mathrm{DMF},(\boldsymbol{\Delta})$ control NP. Each value is represented by the mean $\pm \mathrm{SD}(\mathrm{n}=3) . * * P<0.01 \mathrm{NPB}-\mathrm{DMF}$ vs. NPB and control nanoparticles (NP). ${ }^{\times} P<0.05$ NP vs. NPB-DMF and NPB. 
Figure 6. OVA-specific serum IgG2a and IgG1 in BALB/c mice $(\mathrm{n}=10)$. The animals were immunized on day 0 with a single dose of the formulations containing $20 \mu \mathrm{g}$ OVA for the subcutaneous route $(\mathbf{a}, \mathbf{b})$ or $100 \mu \mathrm{g}$ OVA for the oral route $(\mathbf{c}, \mathbf{d})$. The formulations were: OVA-NPB-DMF $(\boldsymbol{\Delta}), \operatorname{OVA}-\mathrm{NP}(\bullet)$, free OVA $(\Delta)$. AUC is the area under the curve of the immune response profiles calculated over the time of the experiment.

Figure 7. Faecal secretory OVA-specific $\operatorname{IgA}$ in $B A L B / c$ mice $(n=10)$. The animals immunized on day 0 by a single dose of the formulations containing $20 \mu \mathrm{g}$ OVA for the subcutaneous route or $100 \mu \mathrm{g}$ OVA for the oral route. The formulations were: OVANPB-DMF $(\boldsymbol{\Delta})$, OVA-NP $(\bullet)$, free OVA $(\Delta)$. 
$100 \mathrm{mg}$ Gantrez AN dissolved in Incubation of $0.25 \mathrm{mg}$ Vit $B_{12}$ with $12.5 \mathrm{~mL}$ $5 \mathrm{~mL}$ acetone dimethylformamide (DMF), containing $100 \mathrm{mg}$ Gantrez AN for $48 \mathrm{~h}$, at RT
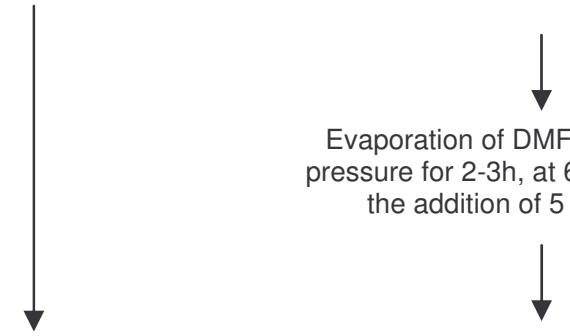

Evaporation of DMF under reduced pressure for $2-3 \mathrm{~h}$, at $60^{\circ} \mathrm{C}$, followed by

the addition of $5 \mathrm{~mL}$ acetone

Gantrez AN nanoparticles formation by the addition of $10 \mathrm{~mL}$ absolute ethanol

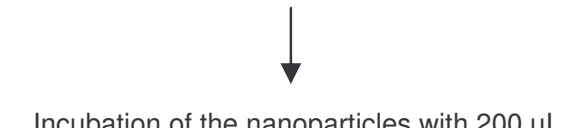

aqueous solution contained 0.5 Vit $\mathrm{B}_{12}$ for $1 \mathrm{~h}$, at

RT.

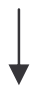

Addition of $3 \mathrm{~mL}$ water, evaporation under reduced pressure, and cross-linking by $100 \mu \mathrm{L}$ 1,3-diaminopropane (1,3-DP)

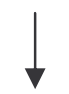

Centrifugation at 27,000 $\times \mathrm{g}$ and then lyophilization using $5 \%$ sucrose<smiles>[CH]1C=CC1</smiles><smiles></smiles>
NPB NPB-DMF

\section{Figure 1}




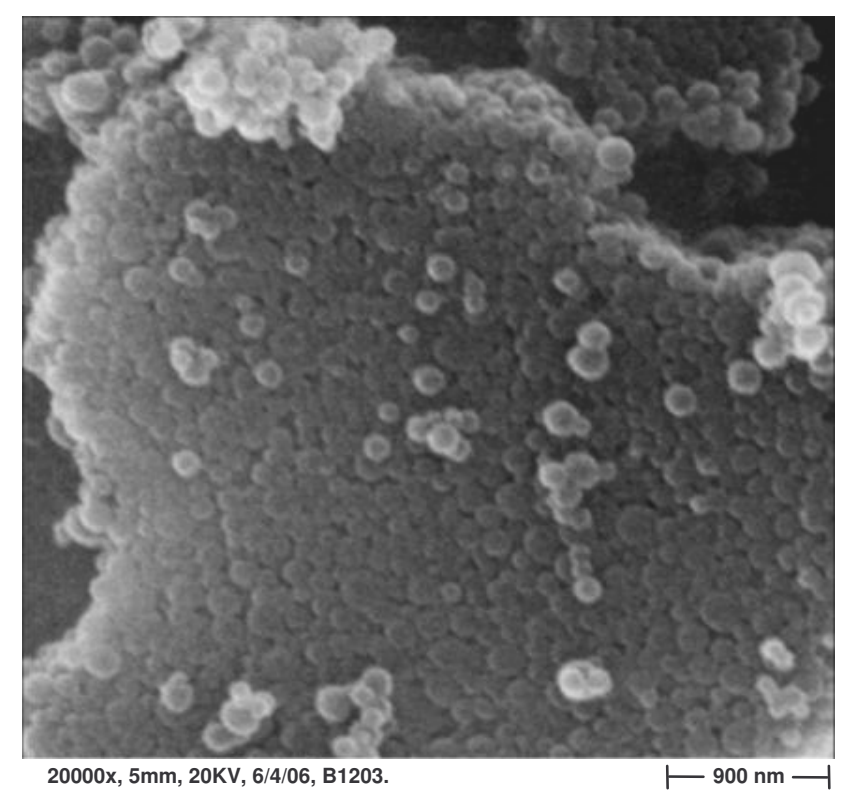

Figure 2 


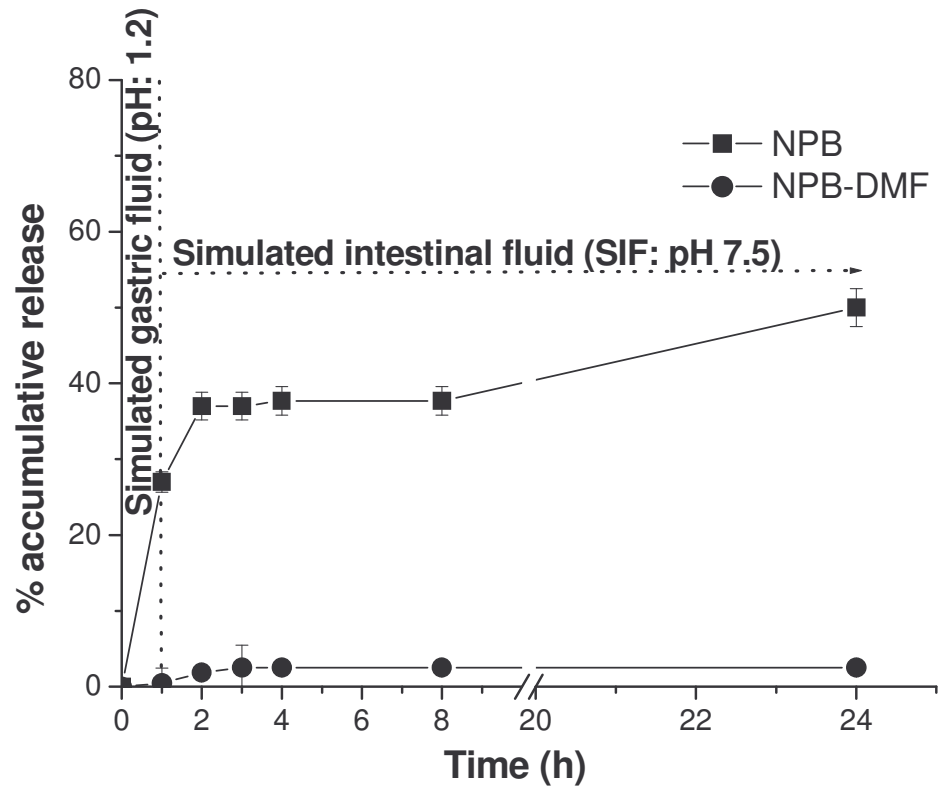

Figure 3 
a

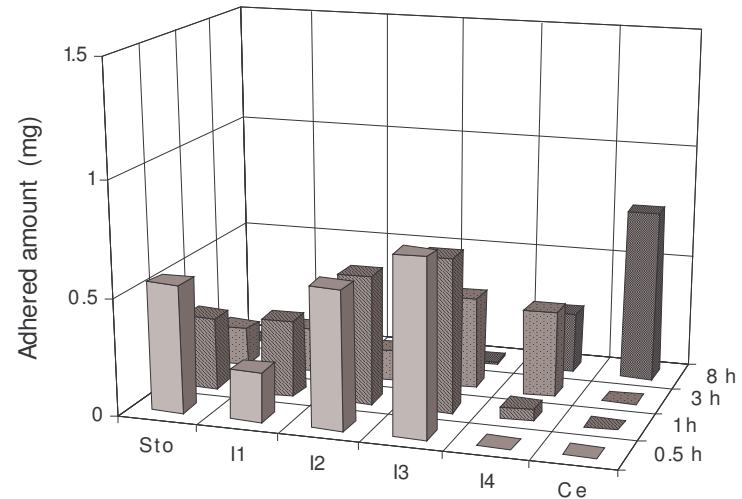

b

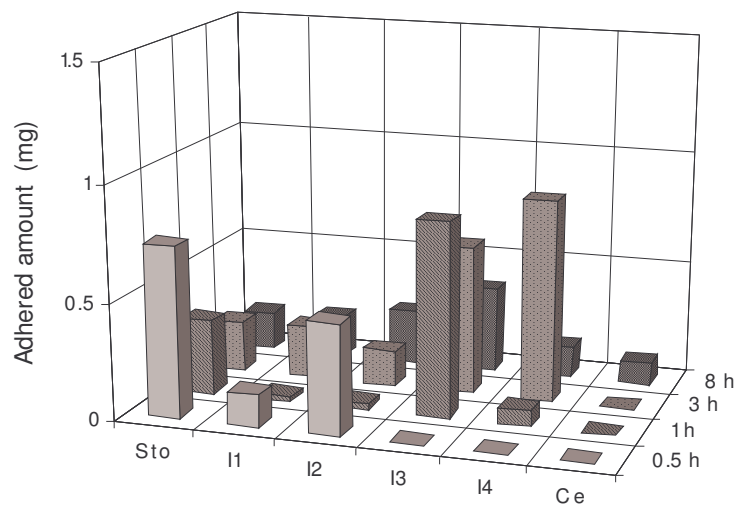

C

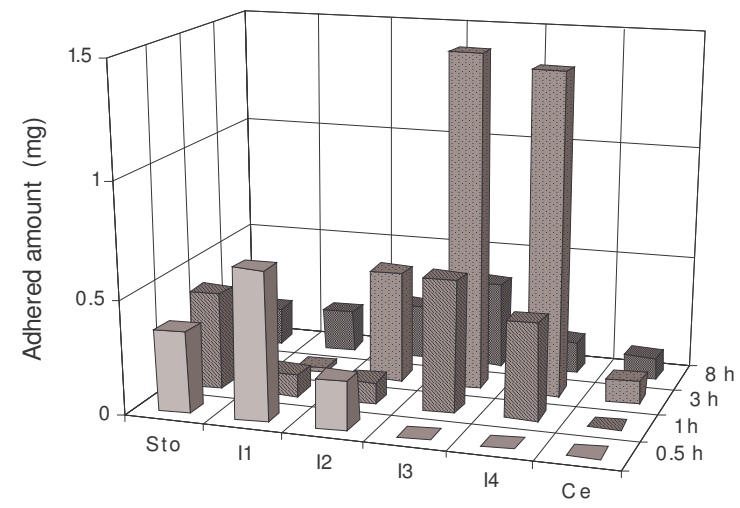

Figure 4 


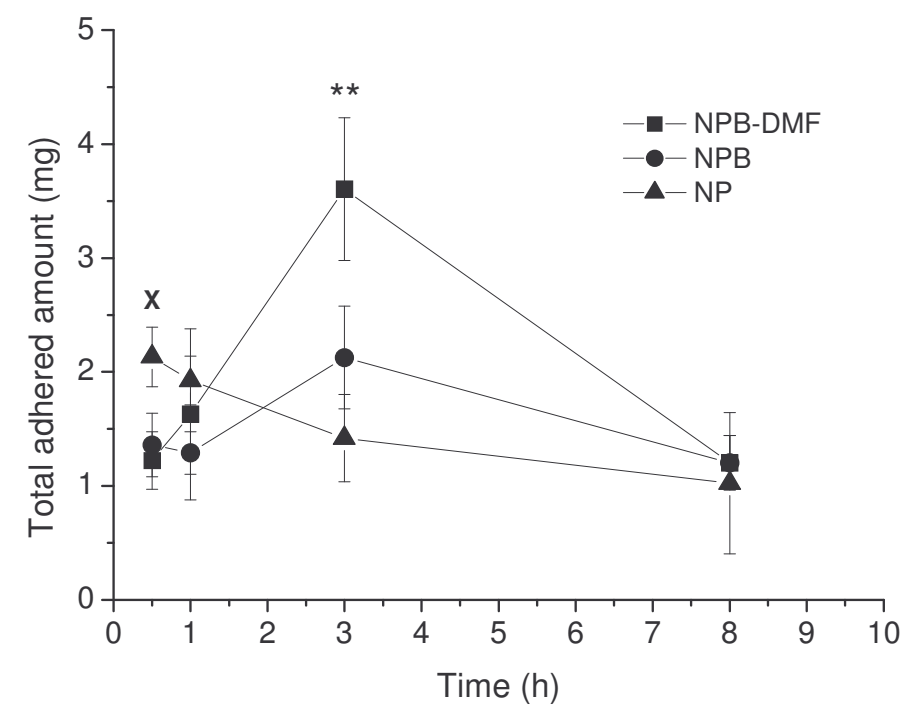

Figure 5 


\section{lgG1 (Th2)}
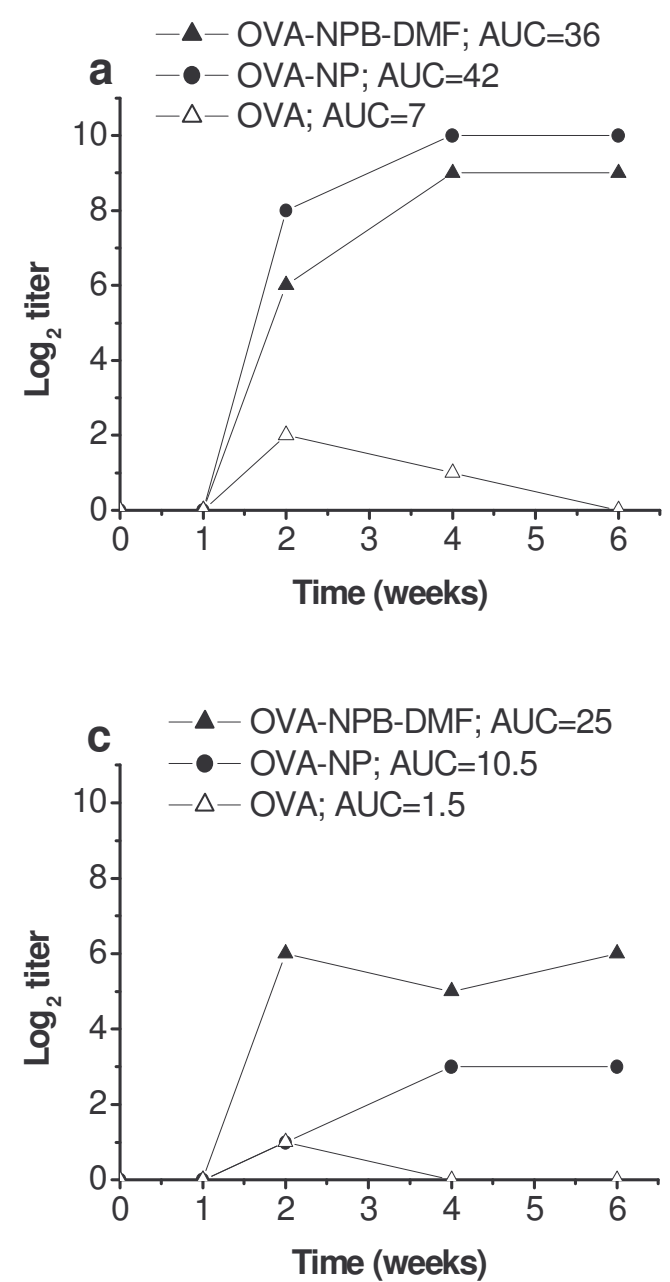

IgG2a (Th1)
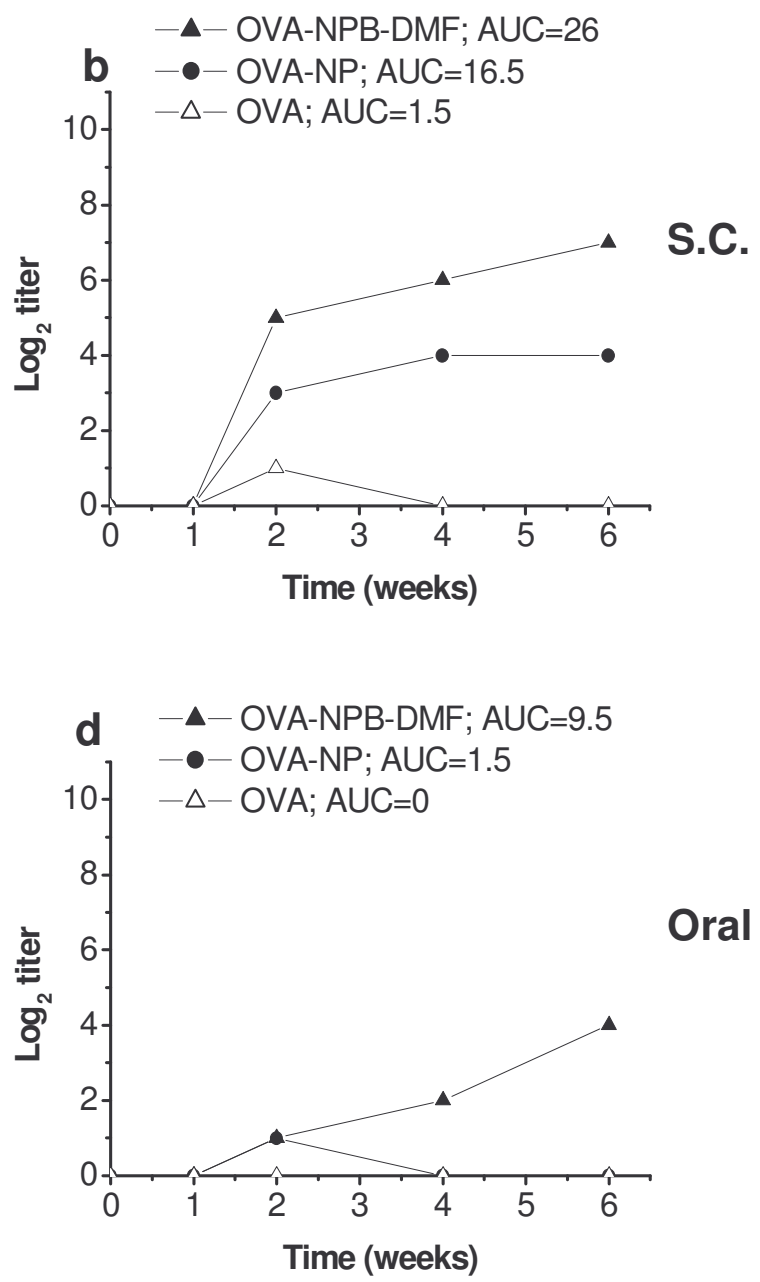

Figure 6 
S.C.

Oral

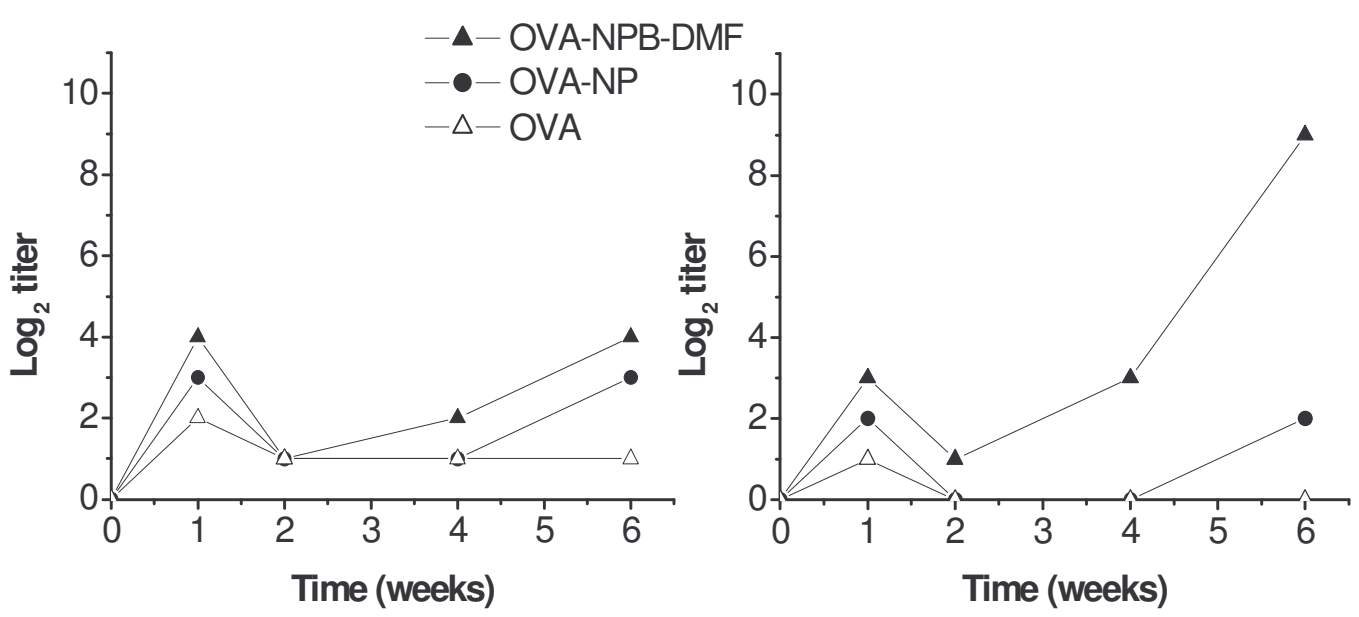

Figure 7 